\title{
Investigation Of Ferroelectric Properties of Rochelle Salt Type Crystals Using A Modified Model
}

\author{
Balkrishna Kandpal * • Mayank Joshi2 • Trilok Chandra Upadhyay2 \\ ${ }^{1}$ Department of Physics, Government College, Jind (Haryana) 126102 \\ ${ }^{2}$ Department of Physics, H.N.B. Garhwal University (A Central University) Srinagar (Garhwal), Uttarakhand-246174
}

*Corresponding Author: kandpalbk@gmail.com

\section{Received: 8.8.2021; Revised: 28.8.2021; Accepted: 19.10.2021}

(OSociety for Himalayan Action Research and Development

\begin{abstract}
Rochelle salt type crystals show ferroelectricity. Previously suggested model is little modified to explain ferroelectric behaviour of three crystals. Simple Green's function approach is used to obtain normal mode frequency formula. This frequency is calculated for different temperatures for Rochelle salt crystal. Theoretical results are compared with others experimental data formulae for dielectric constant and spontaneous polarization are obtained. Values are calculated for Rochelle salt crystal and compared with experimental data of F. Sandy and R. V. Jones (1968).
\end{abstract}

Keyword: Green function • Tangent Loss • Ferroelectricity • Spontaneous Polarization.

\section{Introduction}

Rochelle salt is earliest known ferroelectric crystal. Ferroelectric crystals show spontaneous polarization which is reversible by stress or electric field. Most of ferroelectric crystals have important applications in electronics technology. So, their investigation is useful both from theoretical as well as technological applications.

The Rochelle salt type crystals are Deuterated Rochelle salt, Lithium ammonium tartrate monohydrate (LAT), Tetramethylammonium trichloro mercurate, Lithium thallium tartrate (LTT), Potassium nitrate, Sodium nitrate, Colemanite, Thiourea etc.

Shirane et al (1955) described that Rochelle salt shows ferroelectricity between $255 \mathrm{~K}$ and $297 \mathrm{~K}$. It is orthorhombic in paraelectric and monoclinic in ferroelectric state already investigated by Valasek (1921). Theoretical work has been done earliest by Mueller (1935) from study of Properties of Rochelle salt, Mason (1947), Devonshire (1957) and Mitsui (1958) has given two sub lattice pseudospin model for these crystals. Chaudhuri et al (1980) have added pseudospin-lattice and a fourth-order phonon anharmonic term in this model. Experimentally a large worker has investigated these crystals in recent past are Sandy and Jones (1968), Blinc et al(1964), Akao and Sasaki (1955), Kamba et al(1995), Volkov et al(1985) and Hlinka et al(2001) have particular made dielectric, X-ray analysis, neutron scattering and crystal growth of these crystals. The investigated phase transition properties of Rochelle salt also studied by Rawat and Upadhyay (2019) \& Khan and Upadhyay (2020). A synthesis for 
clarifying phase transition anomalies using In present work, we shell modify Chaudhuri's model ferroelectric nanocomposite from cellulose by adding third order phonon anharmonic terms. We nanoparticles (CNP) and Rochelle salt (RS) at different composition mass ratios performed by Hoai Thuong Nguyen \& Bich Dung Mai(2019). The effect of carbonization on pre-carbonized cellulose nanoparticles at different temperatures on the phase transition and conductive properties of shell use simple Green Function approach to obtain normal mode frequency formula. Dielectric constant and spontaneous electric polarization numerical calculations are done to obtain thermal variations of soft mode frequency, dielectric constant and nanocomposites with Rochelle salt investigated by Hoai Thuong Nguyen (2021).

\section{Theoretical Calculation}

We modify Chuadhari's ${ }^{7}$ model as following -

$$
\begin{aligned}
& H=-2 \Omega \sum_{i}\left(S_{1 i}^{x}+S_{2 i}^{x}\right)-\sum_{i j} J_{i j}\left[\left(S_{1 i}^{z}+S_{2 i}^{z}\right) \quad+\left(S_{1 i}^{z}+S_{2 i}^{z}\right)\right]-\sum_{i j} K_{i j}\left(S_{1 j}^{z} S_{2 i}^{z}\right) \\
& -\Delta \sum_{i}\left(S_{1 i}^{z}+S_{2 i}^{z}\right) \quad-\sum_{i j} V_{i k} S_{1 i}^{z} A_{k}-\sum_{i k} V_{i k} S_{2 i}^{z} A_{k}^{+} \\
& +\frac{1}{4} \sum_{k} \omega_{k}\left(A_{k} A_{k}^{+}+B_{k} B_{k}^{+}\right) \quad+\sum_{k_{1} k_{2} k_{3}} V^{(3)}\left(k_{1}, k_{2}, k_{3}\right) A_{k_{1}} A_{k_{2}} A_{k_{3}} \\
& +\sum_{k_{1} k_{2} k_{3} k_{4}} V^{(4)}\left(k_{1}, k_{2}, k_{3}, k_{4}\right) A_{k_{1}} A_{k_{2}} A_{k_{3}} A_{k 4}
\end{aligned}
$$

where $\Omega$ is proton tunnelling frequency, $S_{z}$ and $S_{x}$ are components of pseudospin variable of $S, V_{i k}$ is spinlattice interaction, $A_{k}$ and $B_{k}$ are positions and momentum operators, $\omega_{k}$ is harmonic phonon frequency $V^{(3)}$ and $\mathrm{V}^{(4)}$ are third-and fourth-order atomic force constants, defined by Born and Huang (1954). $\mathrm{J}_{\mathrm{ij}}$ describes interactions of the diploes fitting to the same and $\mathrm{K}_{\mathrm{ij}}$ to the different sublattices.

Following Zubarev (1960), we consider the evaluation of Green's function (GF)

$$
\begin{gathered}
G_{i j}\left(t-t^{\prime}\right)=\left\langle\left\langle S_{1 i}^{z}(t) ; S_{1 j}^{z}\left(t^{\prime}\right)\right\rangle\right\rangle \\
=-i \theta\left(t-t^{\prime}\right)\left\langle\left[S_{1 i}^{z}(t) ; S_{1 j}^{z}\left(t^{\prime}\right)\right]\right\rangle,
\end{gathered}
$$

Differentiating Eq. (2) twice with respect to time t and t' using the model Hamiltonian (Eq. 1), taking Fourier transformation and applying Dyson's equation, one gets:

$$
G_{i j}(\omega)=G^{0}(\omega)+G^{0}(\omega) \tilde{p}(\omega) G^{0}(\omega)
$$


Where

$$
\begin{array}{r}
G^{0}(\omega)=\frac{\Omega<S_{1 i}^{x}>\delta_{l j}}{\pi\left(\omega^{2}-4 \Omega^{2}\right)} \\
G_{i j}(\omega)=\frac{G^{0}(\omega)}{\left[1-G^{0}(\omega) p(\omega)\right]},
\end{array}
$$

and

$$
\begin{aligned}
& \widetilde{P}(\omega)=\frac{\pi i\left\langle\left[F(t), S_{1 j}^{y}\right]\right\rangle}{\Omega\left\langle S_{1 i}^{x}\right\rangle^{2}} \\
& +\frac{\pi^{2}}{\Omega^{2}\left\langle S_{1 i}^{x}\right\rangle^{2}}\left\langle\left\langle F_{i}(t) ; F_{j}^{\prime}\left(t^{\prime}\right)\right\rangle\right\rangle,
\end{aligned}
$$

and

$$
\begin{aligned}
& F\left(t^{\prime}\right)=2 \Omega J_{i j}\left(S_{1 i}^{x} S_{1 i}^{z}+S_{1 i}^{z} S_{1 i}^{x}\right)-2 \Omega K_{i j}\left(S_{1 i}^{x} S_{2 i}^{z}\right) \\
& +2 \Omega V_{i k} S_{1 i}^{x} A_{k}+2 \Omega \Delta\left(S_{1 i}^{x}+S_{2 i}^{z}\right)+2 \Omega V_{i k} A_{k}^{+} S_{2 i}^{x}
\end{aligned}
$$

The Green's Function (GF), Eq. (5) can be written as:

$$
G(\omega)=\frac{\Omega\left\langle S_{1 i}^{x}\right\rangle \delta_{i j}}{\pi\left[\omega^{2}-\widehat{\Omega}^{2}-\widetilde{P}(\omega)\right]}
$$

where renormalized frequency $\widehat{\Omega}$, in lowest approximation is given as:

$$
\widehat{\Omega}^{2}=4 \Omega^{2}+\frac{i}{\left\langle S_{1 i}^{x}\right\rangle}\left\langle\left[F, S_{1 i}^{y}\right]\right\rangle
$$

and polarization operator $\quad \widetilde{\widetilde{P}}(\omega)$ is given as :

$$
\widetilde{\widetilde{P}}(\omega)=\frac{\pi}{\Omega\left\langle S^{x}\right\rangle}\left\langle\left\langle\left[F_{i}(t) ;\right] F_{j}^{\prime}\left(t^{\prime}\right)\right\rangle\right\rangle
$$

so that 


$$
\widehat{\Omega}^{2}=a^{2}+b^{2}-b c
$$

with

$$
a=2 J_{0}\left\langle S_{1}^{x}\right\rangle+K_{0}\left\langle S_{2}^{x}\right\rangle+\Delta
$$

$$
b=2 \Omega
$$

and

$$
c=2 J\left\langle S_{1}^{x}\right\rangle+K\left\langle S_{2}^{x}\right\rangle
$$

Putting values of $\widetilde{\widetilde{P}}(\omega)$ in Eq. (8), Green's function finally becomes:

$$
G(\omega)=\frac{\Omega\left\langle S_{1 i}^{x}\right\rangle \delta_{i j}}{\pi\left[\omega^{2}-\widehat{\Omega}^{2}-2 i \Omega \Gamma(\omega)\right]}
$$

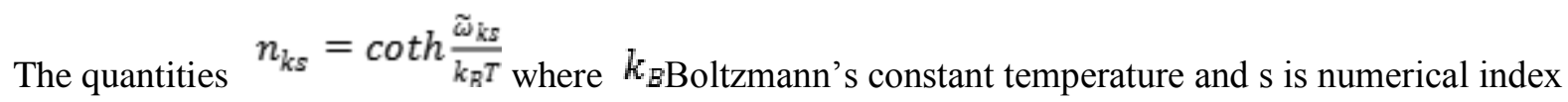
$\mathrm{s}=1,2,3,4$ : the frequency $\widehat{\Omega}$ is given by:

$$
\widehat{\Omega}^{2}=\widetilde{\Omega}^{2}+2 \Omega \Delta_{s-p}(\omega)
$$

and

$$
\widetilde{\Omega}^{2}=\widetilde{\Omega}^{2}+2 \Omega \Delta_{s}(\omega)
$$

\subsection{Soft Mode Frequency and Transition Temperatures}

Solving Eq. (10) self consistently, one obtains renormalized frequency:

$$
\widehat{\Omega}_{-}{ }^{2}=\frac{1}{2}\left[\left(\widetilde{\omega}_{k}^{2}+\widetilde{\Omega}^{2}\right) \pm\left\{\left(\widetilde{\omega}_{k}^{2}-\widetilde{\Omega}^{2}\right)^{2}+8 V_{i k}^{2}\left\langle S^{x}\right)\right\}^{\frac{1}{2}} \Omega\right]
$$

The frequency $\widehat{\Omega}$ is the soft mode frequency which critically depends on temperature and is responsible for phase transition. 


\subsection{Dielectric Constant and Loss Tangent}

The expression for dielectric constant can be expressed as $\varepsilon(\omega)\rangle\rangle 1$ is following:

$$
\varepsilon(\omega) 1=-8 \pi N \mu^{2}\left\langle S_{1}^{x}\right\rangle\left(\omega^{2}-\widehat{\Omega}^{2}\right)\left[\left(\omega^{2}-\widehat{\Omega}^{2}\right)^{2}+4 \Omega^{2} \Gamma^{2}(\omega)\right]^{1}
$$

\subsection{Spontaneous Polarization}

The Spontaneous Polarization ( $P S$ ) is given by Halblutzel ${ }^{16}$

$$
P s=2 N \mu\left(\left\langle S_{1}^{z}\right\rangle+\left\langle S_{2}^{z}\right\rangle\right)
$$

Putting the values of $N \mu$ and $\left\langle S_{1}^{z}\right\rangle$ and $\left\langle S_{2}^{z}\right\rangle$ we obtain the value of $P s$ for Rochelle salt. We compare our results with Sandy and Jones (1968). A good agreement is obtained.

\section{Numerical Calculation and Results}

By using model values of Physical quantities, the thermal variations of soft mode frequency, dielectric constant and spontaneous polarization are shown in Fig. 1, 2 and 3. Fig 1. reveals that the electrical permittivity first increases by increasing the temperature and then decreases and reaches a minimum value at $0 \mathrm{~K}$ and follow a same pattern for further temperature. Fig 2. Shows that soft mode frequency increases with increasing temperature and reaches a maximum value near transition temperature, after that decreases and then slightly increases which strictly follow the Cochran's behaviour. Fig 3. Shows that the spontaneous polarization first increases by increasing temperature and reaches a maximum value 2.5 near transition temperature and then decreases. The constant values given by Chaudhuri et al $(1980) \mathrm{T}_{\mathrm{c} 1}=255-2 \mathrm{~K}, \mathrm{~T}_{\mathrm{c} 2}=296.9 \mathrm{~K}, \mathrm{C}_{1}=1830 \mathrm{~K}, \mathrm{C}_{2}=2248 \mathrm{~K}, \eta=5.51 \mathrm{~cm}^{-1}, \Delta=0.678 \mathrm{~cm}^{-1}, \Omega^{2}$

$$
\begin{gathered}
\left(\mathrm{J}^{\prime}+\mathrm{K}^{\prime}\right) *=2738 \mathrm{~cm}^{-3}, \Omega^{2} \quad(\mathrm{~J}+\mathrm{K})=2340 \quad \mathrm{~cm}^{-1}, \Delta=0.678 \quad \mathrm{~cm}^{-1}, \quad \omega_{k}^{2}=520_{\mathrm{cm}^{-1}}, \\
\Omega V_{i k}=20.92 \mathrm{~K} \\
\quad, \mathrm{~A}_{0} \mathrm{k}_{\mathrm{B}} \mathrm{X} 10^{17}=5.737 \mathrm{prg} / \mathrm{K}, \mathrm{N}=3.8 \times 10^{21} \mathrm{~cm}^{-3}, \mu=1.51 \times 10^{18} \text { esu. }
\end{gathered}
$$

The temperature dependence of $\left\langle S_{1}^{z}\right\rangle,\left\langle S_{2}^{z}\right\rangle,\left\langle S_{1}^{x}\right\rangle,\left\langle S_{2}^{x}\right\rangle, \widetilde{\Omega}, \widetilde{\Omega}, \widehat{\Omega}$ and permittivity (E) across Tc have been calculated. 


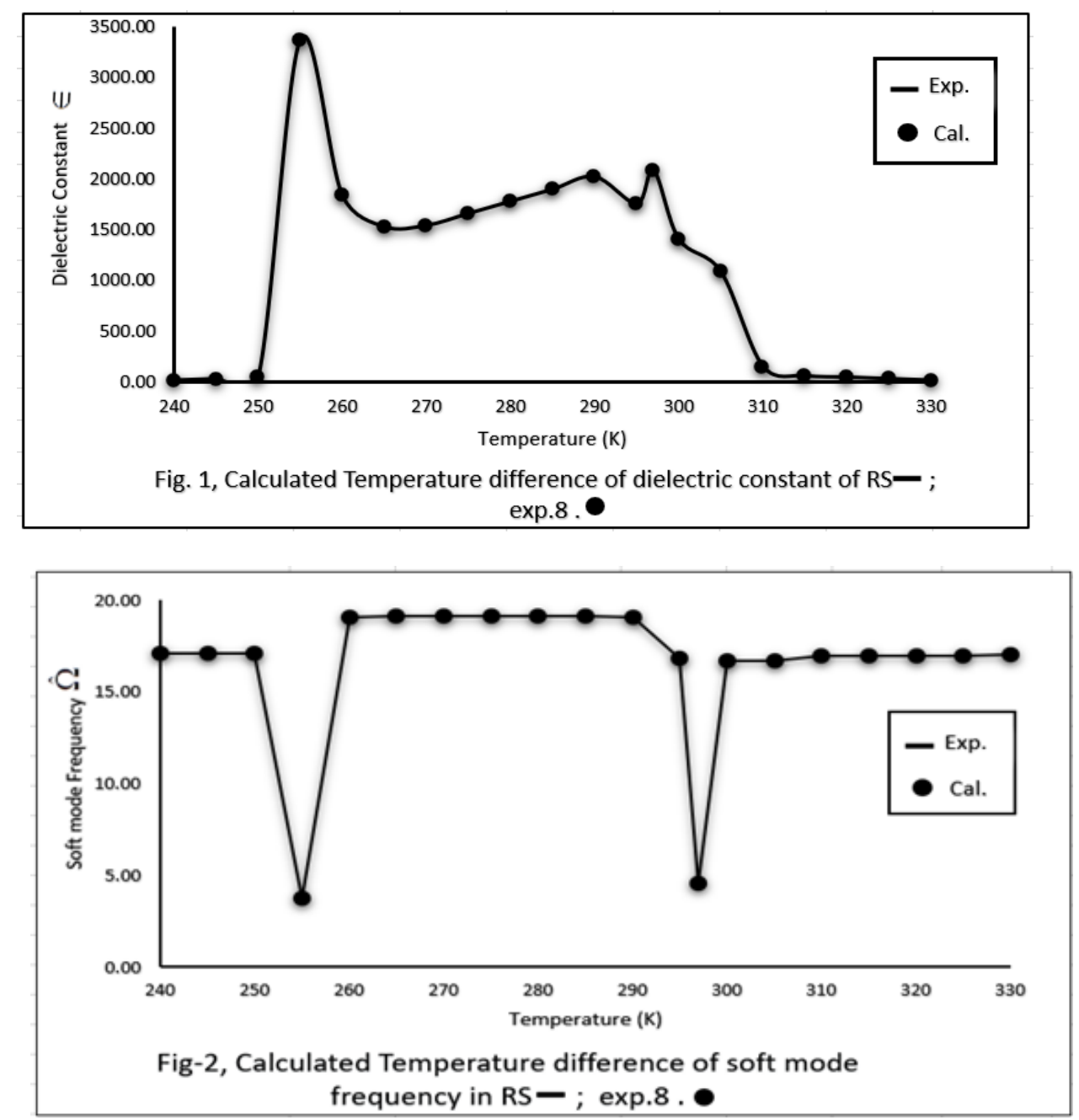

Table 1- Calculated temperature dependence of Spontaneous Polarization in Rochelle salt

\begin{tabular}{cc}
$\mathbf{T}(\mathbf{K})$ & $P s \times 10^{7}\left(c / \mathrm{cm}^{2}\right)$ \\
\hline 250 & 0.0 \\
255 & 0.0 \\
260 & 1.5 \\
265 & 2.0 \\
270 & 2.4 \\
275 & 2.5 \\
280 & 2.4 \\
285 & 2.2 \\
290 & 2.0 \\
295 & 1.5 \\
297 & 0.0 \\
300 & 0.0
\end{tabular}

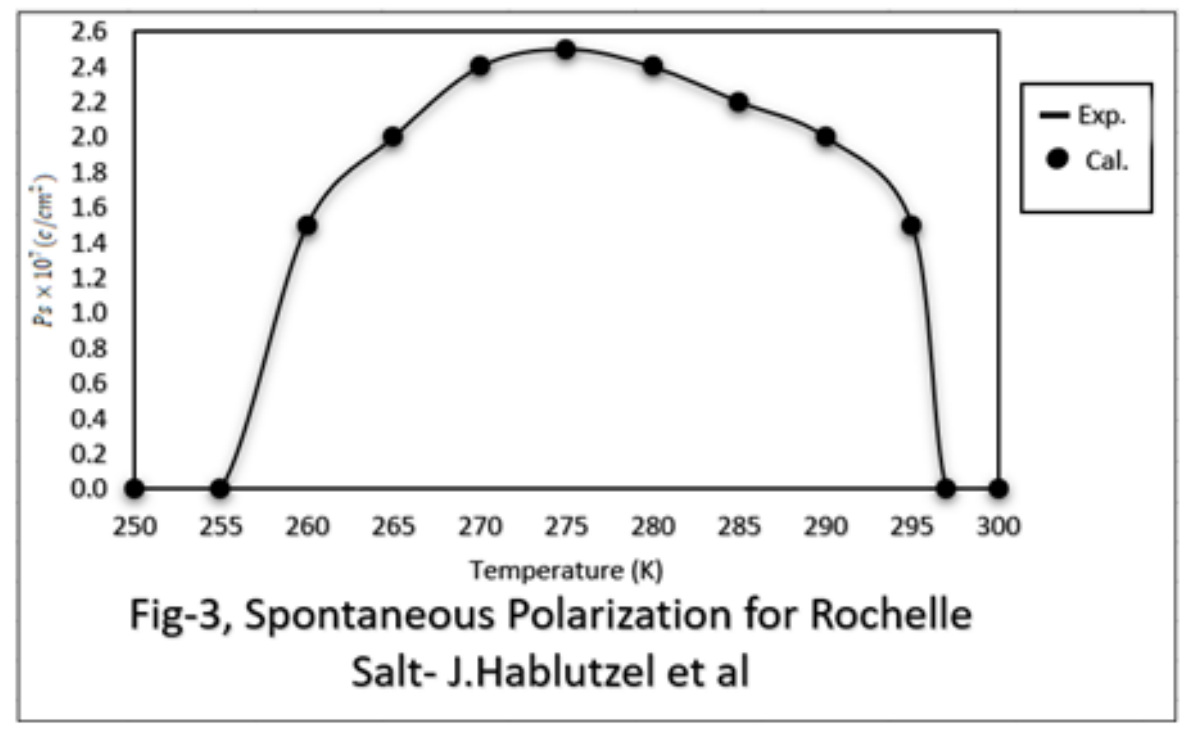




\section{Conclusion}

In this paper, present work reveals that the two-sublattice PLCM model along with a thirdand fourth-order phonon anharmonic interaction terms. These extra interaction terms play an important role to explain well the thermal dependence physical quantities of soft mode frequency, dielectric constant and spontaneous polarization in Rochelle salt crystal.

\section{Acknowledgement}

The authors are thankful Prof. S.C. Bhatt, Prof. P.D. Semalty, Prof. Mahavir Singh (Shimla), Prof. L.P. Purohit (Haridwar), Prof. R.K. Shukla (Lucknow), Prof.N.S. Negi (Shimla)and Dr. Manish Uniyal for their kind encouragement and Mr. Kuldeep Singh (Research Scholar) for help.

\section{References}

Akao, H., \& Sasaki, T. (1955). Dielectric Dispersion of Rochelle Salt in the Microwave Region. The Journal of Chemical Physics, 23(12), 22102214. doi:10.1063/1.1740725

Blinc, R., Petkovšek, J., \& Zupančič, I. (1964). $\mathrm{Na}^{23}$ Magnetic-Resonance Study of the Ferroelectric Transition in Rochelle Salt. Physical Review, 136(6A), A1684A1692. doi:10.1103/physrev.136.a1684

Born, M. and Huang, K. Dynamical Theory of CrystalLattices (Oxford Univ ersity Press, NewYork, 1954). DOI:10.1119/1.1934059

Chaudhuri B. K., Atake, T., Ganguli, S., \& Chihara, H. (1980). Study of Ferroelectric Phase Transitions in the Rochelle Salt with the Pseudo-SpinLattice Coupled Mode Model. Journal of the Physical Society of Japan, 49(2), 608-618. doi:10.1143/jpsj.49.608
Devonshire, A. F. (1957). Theory of Rochelle salt. Philosophical Magazine, 2(20), 1027-1039. doi:10.1080/14786435708238209

Hablützel, Jakob, Doctoral Thesis on Heavy Seignette salt, dielectric investigations on $\mathrm{KNaC}_{4} \mathrm{H}_{4} \mathrm{O}_{6} \cdot 4 \mathrm{H}_{2} \mathrm{O} \quad$ crystals (1939), doi:10.3929/ethz-a-000098905

Hlinka,J., Kulda, J., Kamba, S., and Petzelt, J. (2001), Resonant soft mode in Rochelle salt by inelastic neutron scattering, Physical Review B, 63, 052102 doi:10.1103/PhysRevB.63.052102

Hoai Thuong Nguyen \& Bich Dung Mai (2019), Study on structure and phase transition of an ecofriendly ferroelectric composite prepared from cellulose nanoparticles mixed with Rochelle salt, Vol. 92(9) 831-838. doi:10.1080/01411594.2019.1650931

Hoai Thuong Nguyen (2021), Influence of carbonization on phase transition and electrical conductivity of ferroelectric composite with Rochelle salt inclusion, Ferroelectrics, Vol 572(1) 221228. doi:10.1080/00150193.2020.1868884

Kamba, S., Schaack, G., \& Petzelt, J. (1995). Vibrational spectroscopy and soft-mode behavior in Rochelle salt. Physical Review B, 51(21), 14998-15007. doi:10.1103/physrevb.51.14998

Khan, Muzaffar Iqbal \& Upadhyay, Trilok Chandra, Study of phase transition properties in Rochelle salt crystal, AIR Vol.02(1), 28-31 [March 2020],

http://nopr.niscair.res.in/handle/123456789/549 $\underline{65}$

Mason, W. P. (1947). Theory of the Ferroelectric Effect and Clamped Dielectric Constant of Rochelle Salt. Physical Review, 72(9), 854865. doi: $10.1103 /$ physrev. 72.854 
345.

Mitsui, T. (1958), Theory of the Ferroelectric doi:10.1070/PU1960v003n03ABEH003275 Effect in Rochelle salt, Physical Review 111(5), 1259 doi:10.1103/PhysRev.111.1259

Mueller, H. (1935). Properties of Rochelle salt. Physical Review, 47(2), 175-191. $\underline{10.1103 / P h y s R e v .47 .175}$

Rawat, Aanchal \& Upadhyay, Trilok Chandra (2019), Temperature dependence of soft mode frequency, dielectric constant and loss tangent of deuterated Rochelle salt crystal, Indian Journal of Pure \& Applied Physics Vol. 57,144146

http://nopr.niscair.res.in/handle/1234567 $\underline{89 / 45799}$

Sandy, F., \& Jones, R. V. (1968). Dielectric Relaxation of Rochelle Salt. Physical Review, 168(2), 481- 493.

doi:10.1103/physrev.168.481

Shirane, G., Jona, F., \& Pepinsky, R. (1955). Some Aspects of Ferroelectricity. Proceedings of the IRE, 43(12), 1738 1793. doi:10.1109/jrproc. 1955.278041

Valasek, J. (1921). Piezo-Electric and Allied Phenomena in Rochelle Salt. Physical Review, 17(4), 475- 481. doi:10.1103/physrev.17.475

Volkov, A. A., Goncharov, Y. G., Kozlov, G. V., Kryukova, E. B., \& Prokhorov, A. M. (1985). Transformation of the relaxational soft mode into an optical phonon in Rochelle salt. Ferroelectrics, 63(1), 197-206. doi:10.1080/00150198508221401

Zubarev, D. N. (1960). Double-Time Green Functions in Statistical Physics. Soviet Physics Uspekhi, 3(3), 320- 